\title{
On Positive Integer Powers of Toeplitz Matrices
}

\author{
Honglin $\mathrm{Wu}^{1}$ \\ ${ }^{1}$ Department of Mathematics, Shanghai Business School, Shanghai, P. R. China \\ Correspondence: Honglin Wu, Department of Mathematics, Shanghai Business School, 123 Fengpu Ave., Fengx- \\ ian District, Shanghai 201400, P. R. China. E-mail: mathswu@163.com
}

Received: October 8, 2013 Accepted: October 30, 2013 Online Published: November 14, 2013

doi:10.5539/jmr.v5n4p52 URL: http://dx.doi.org/10.5539/jmr.v5n4p52

The research is supported by Excellent Young Teacher Foundation of Shanghai (sxy10004) and by Foundations of Shanghai Business School for teachers with a Doctor's degree, A-0201-00-050-205, BN2154-3-1

\begin{abstract}
In this paper, we restate a necessary and sufficient condition for all positive integer powers of a Toeplitz matrix to be still Toeplitz matrices which was ever studied by T. N. E. Greville (1983) and Tamir Shalom (1987), and our new proof is clear and self-contained. We also derive some identities involving the entries of the Toeplitz matrix and those of its positive integer powers in case that the powers are still Toeplitz matrices.
\end{abstract}

Keywords: Toeplitz matrix, positive integer power, main diagonal, upper- $r$ th diagonal, lower- $r$ th diagonal

AMS Subject Classifications: 15A23, 05B20, 65F99

\section{Introduction}

A Toeplitz matrix is an $n \times n$ matrix $A=\left(a_{i j}\right)$ where $a_{i j}=a_{j-i}$, i.e., a matrix of the form

$$
A=\left(\begin{array}{ccccc}
a_{0} & a_{1} & a_{2} & \cdots & a_{n-1} \\
a_{-1} & a_{0} & a_{1} & \cdots & a_{n-2} \\
a_{-2} & a_{-1} & a_{0} & \ddots & \vdots \\
\vdots & \vdots & \ddots & \ddots & a_{1} \\
a_{-n+1} & a_{-n+2} & \cdots & a_{-1} & a_{0}
\end{array}\right),
$$

where $a_{-n+1}, a_{-n+2}, \ldots, a_{-1}, a_{0}, a_{1}, a_{2}, \ldots, a_{n-1} \in \mathbb{C}$. The entries of $A$ are constant down the diagonals parallel to the main diagonal. Toeplitz matrix arises in scientific computing and engineering, for example, image processing, numerical differential equations and integral equations, time series analysis and control theory (see Chan \& $\mathrm{Ng}$, 1996; Gray, 2006; Horn \& Johnson, 1985; Kailath \& Sayed, 1995). A great deal is known about the behavior of such matrices-the most common and complete references being Grenander and Szegö (1958). A more recent text devoted to the subject is Böttcher and Silbermann (1999).

For an $n \times n$ matrix $A=\left(a_{i j}\right)$, we call the diagonal parallel to the main diagonal which has the entries $a_{i, i+r}(i=$ $1,2, \ldots, n-r)$ the upper-rth diagonal for $r=1,2, \ldots, n-1$, and call the diagonal parallel to the main diagonal which has the entries $a_{i, i-r}(i=r+1, r+2 \ldots, n)$ the lower-rth diagonal for $r=1,2, \ldots, n-1$. Given an $n \times n$ Toeplitz matrix $A=\left(a_{i j}\right)=\left(a_{j-i}\right)$, the upper-rth diagonal has the constant entry $a_{r}$, and the lower- $r$ th diagonal has the constant entry $a_{-r}$ for $r=1,2, \ldots, n-1$.

Notice that the positive integer powers of Toeplitz matrices are not necessarily Toeplitz matrices. However, we can still find a great deal of Toeplitz matrices whose positive integer powers are Toeplitz matrices, for example, circulant matrices. So what additional conditions do those kinds of Toeplitz matrices have? In section 2, we restate a necessary and sufficient condition for Toeplitz matrices to have Toeplitz positive integer powers which was ever studied before. Moreover, we also get some additional results about this problem In section 3, we give some examples to interpret our result. 


\section{Main Results}

T. N. E. Greville obtained a criterion for a nonsingular Toeplitz matrix to have a Toeplitz inverse in 1983 (see Greville, 1983). In 1987, Tamir Shalom derived an equivalent necessary and sufficient condition for a nonsingular Toeplitz matrix to have a Toeplitz inverse, and proved that the statement "All positive powers of a nonsingular Toeplitz matrix are Toeplitz matrices" is equivalent to the statement "The inverse of a nonsingular Toeplitz matrix is a Toeplitz matrix" (see Shalom, 1987). We restate the necessary and sufficient condition for Toeplitz matrices to have Toeplitz positive integer powers and give a new proof.

Obviously, any power of a Toeplitz matrix of order $n=1$ is naturally a Toeplitz matrix, and any power of a scalar matrix which is a diagonal matrix whose diagonal elements are the same scalar is also a scalar matrix that is naturally a Toeplitz matrix. So we only need to consider Toeplitz matrices of order $n \geq 2$ which are not scalar matrices in the following. Now we derive an important result for the product of two Toeplitz matrices which are not scalar matrices to be still a Toeplitz matrix, and obtain some connections between the entries of the two Toeplitz matrices and those of their product.

Lemma 1 Let $A=\left(a_{j-i}\right)_{i, j=1}^{n}, B=\left(b_{j-i}\right)_{i, j=1}^{n}$ be Toeplitz matrices which are not scalar matrices, $n \geq 2$. If $a_{i} b_{j-n}=a_{i-n} b_{j}$ for $i, j=1, \ldots, n-1$, then the product $A B$ is also a Toeplitz matrix. Furthermore, if we let $A B=C=\left(c_{j-i}\right)_{i, j=1}^{n}$, then $c_{i} a_{j-n}=c_{i-n} a_{j}$ and $c_{i} b_{j-n}=c_{i-n} b_{j}$ for $i, j=1, \ldots, n-1$.

Proof. Suppose $A=\left(a_{i j}\right)_{i, j=1}^{n}=\left(a_{j-i}\right)_{i, j=1}^{n}, B=\left(b_{i j}\right)_{i, j=1}^{n}=\left(b_{j-i}\right)_{i, j=1}^{n}$ are Toeplitz matrices which are not scalar matrices, and $A B=C=\left(c_{i j}\right)_{i, j=1}^{n}$. Then $c_{i j}=\sum_{p=1}^{n} a_{i, p} b_{p, j}=\sum_{p=1}^{n} a_{p-i} b_{j-p}$ for $1 \leq i, j \leq n$. Thus, for $r=1,2, \ldots, n-1$, the entries of $C$ down the upper-rth diagonal are

$$
c_{i, i+r}=\sum_{p=1}^{n} a_{p-i} b_{i+r-p}=\sum_{q=1-i}^{n-i} a_{q} b_{r-q}, i=1, \cdots, n-r ;
$$

the entries of $C$ down the lower- $(n-r)$ th diagonal are

$$
c_{i, i+r-n}=\sum_{p=1}^{n} a_{p-i} b_{i+r-n-p}=\sum_{q=1-i}^{n-i} a_{q} b_{r-n-q}, i=n-r+1, \cdots, n .
$$

And the entries of $C$ on the main diagonal are

$$
c_{i, i}=\sum_{p=1}^{n} a_{p-i} b_{i-p}=\sum_{q=1-i}^{n-i} a_{q} b_{-q}, i=1,2, \cdots, n .
$$

Now we assume $a_{i} b_{j-n}=a_{i-n} b_{j}$ for $i, j=1, \ldots, n-1$.

Case (I) $a_{i} a_{i-n}=0$ for any $i=1, \ldots, n-1$.

Then for any $j=1, \ldots, n-1$, we have $0=a_{i} b_{j-n} a_{i-n} b_{j}=a_{i}^{2} b_{j-n}^{2}=a_{i-n}^{2} b_{j}^{2}$. That is, $a_{i} b_{j-n}=a_{i-n} b_{j}=0$ for any $i, j=1, \ldots, n-1$.

Subcase (I) There exists an $i_{0}\left(1 \leq i_{0} \leq n-1\right)$ such that $a_{i_{0}}=0$ and $a_{i_{0}-n} \neq 0$.

Since $a_{i_{0}-n} b_{j}=a_{i_{0}} b_{j-n}=0$, we have $b_{j}=0(j=1, \ldots, n-1)$, i.e., $B$ is a lower triangular Toeplitz matrix. Considering that $B$ is not a scalar matrix, so there exists an $j_{0}\left(1 \leq j_{0} \leq n-1\right)$ such that $b_{j_{0}-n} \neq 0$. Then $a_{i}=0(i=1, \ldots, n-1)$ since $a_{i} b_{j_{0}-n}=a_{i-n} b_{j_{0}}=0$, i.e., $A$ is also a lower triangular Toeplitz matrix. Thus we only need to show that the lemma holds for the case in which $A$ and $B$ are both lower triangular Toeplitz matrices. Note that, for $r=1,2, \ldots, n-1$, the entries of $C$ down the upper-rth diagonal are

$$
c_{i, i+r}=\sum_{q=1-i}^{n-i} a_{q} b_{r-q}=0, i=1, \cdots, n-r ;
$$

the entries of $C$ down the lower- $(n-r)$ th diagonal are

$$
c_{i, i+r-n}=\sum_{q=1-i}^{n-i} a_{q} b_{r-n-q}=\sum_{q=r-n}^{0} a_{q} b_{r-n-q},
$$


for all $i=n-r+1, \cdots, n$. And the entries of $C$ on the main diagonal are

$$
c_{i, i}=\sum_{q=1-i}^{n-i} a_{q} b_{-q}=a_{0} b_{0}, i=1,2, \cdots, n .
$$

Thus, $C=A B$ is also a lower triangular Toeplitz matrix. Furthermore, if we let $A B=C=\left(c_{j-i}\right)_{i, j=1}^{n}$, then $c_{i} a_{j-n}=0=c_{i-n} a_{j}$ and $c_{i} b_{j-n}=0=c_{i-n} b_{j}$ for $i, j=1, \ldots, n-1$.

Subcase (II) There exists an $i_{0}\left(1 \leq i_{0} \leq n-1\right)$ such that $a_{i_{0}} \neq 0$ and $a_{i_{0}-n}=0$.

This subcase can be proved similarly to Subcase (I). In fact, $A$ and $B$ are both upper triangular Toeplitz matrices in this subcase.

Case (II) $b_{i} b_{i-n}=0$ for any $i=1, \ldots, n-1$.

This case can be proved similarly to Case (I).

Case (III) There exist $p, q(1 \leq p, q \leq n-1)$ such that $a_{p} a_{p-n} \neq 0$ and $b_{q} b_{q-n} \neq 0$.

Considering that $b_{i} a_{p-n} b_{j-n} a_{p}=b_{i-n} a_{p} b_{j} a_{p-n}$ and $a_{i} b_{q-n} a_{j-n} b_{q}=a_{i-n} b_{q} a_{j} b_{q-n}$ for $i, j=1, \ldots, n-1$, we have $b_{i} b_{j-n}=b_{i-n} b_{j}$ and $a_{i} a_{j-n}=a_{i-n} a_{j}$ for $i, j=1, \ldots, n-1$.

Since $a_{i} b_{j-n}=a_{i-n} b_{j}$ for $i, j=1, \ldots, n-1$, we get equations for $r=0,1,2, \cdots, n-2$ :

$$
\begin{aligned}
a_{n-1} b_{r-n+1} & =a_{-1} b_{r+1}, \\
a_{n-2} b_{r-n+2} & =a_{-2} b_{r+2}, \\
& \cdots \\
a_{r+1} b_{-1} & =a_{r-n+1} b_{n-1} .
\end{aligned}
$$

Hence,

$$
\begin{aligned}
& \sum_{q=0}^{n-2} a_{q} b_{r-q}+a_{n-1} b_{r-n+1}=a_{-1} b_{r+1}+\sum_{q=0}^{n-2} a_{q} b_{r-q}, \\
& \sum_{q=-1}^{n-3} a_{q} b_{r-q}+a_{n-2} b_{r-n+2}=a_{-2} b_{r+2}+\sum_{q=-1}^{n-3} a_{q} b_{r-q}, \\
& \sum_{q=r-n+2}^{r} a_{q} b_{r-q}+a_{r+1} b_{-1}=a_{r-n+1} b_{n-1}+\sum_{q=r-n+2}^{r} a_{q} b_{r-q} .
\end{aligned}
$$

That is,

$$
\begin{gathered}
c_{1,1+r}=\sum_{q=0}^{n-1} a_{q} b_{r-q}=\sum_{q=-1}^{n-2} a_{q} b_{r-q}=c_{2,2+r}, \\
c_{2,2+r}=\sum_{q=-1}^{n-2} a_{q} b_{r-q}=\sum_{q=-2}^{n-3} a_{q} b_{r-q}=c_{3,3+r}, \\
\ldots \quad \ldots \quad \ldots \\
c_{n-r-1, n-1}=\sum_{q=r-n+2}^{r+1} a_{q} b_{r-q}=\sum_{q=r-n+1}^{r} a_{q} b_{r-q}=c_{n-r, n} .
\end{gathered}
$$

Therefore, the entries down the upper- $r$ th diagonal of $C$ are equal for $r=1,2, \ldots, n-1$ and the entries on the main diagonal of $C$ are also equal.

Similarly, we get equations for $r=2,3, \cdots, n-1$ (suppose $n \geq 3$ now without loss of generality since it is not necessary to prove the case $n=2$ here):

$$
\begin{aligned}
a_{r-1} b_{1-n} & =a_{r-n-1} b_{1}, \\
a_{r-2} b_{2-n} & =a_{r-n-2} b_{2}, \\
& \cdots \\
a_{1} b_{r-n-1} & =a_{1-n} b_{r-1} .
\end{aligned}
$$


So

$$
\begin{array}{rlll}
\sum_{q=r-n}^{r-2} a_{q} b_{r-n-q}+a_{r-1} b_{1-n} & = & a_{r-n-1} b_{1}+\sum_{q=r-n}^{r-2} a_{q} b_{r-n-q}, \\
\sum_{q=r-n-1}^{r-3} a_{q} b_{r-n-q}+a_{r-2} b_{2-n} & = & a_{r-n-2} b_{2}+\sum_{q=r-n-1}^{r-3} a_{q} b_{r-n-q}, \\
\ldots & \ldots & \ldots \\
\sum_{q=2-n}^{0} a_{q} b_{r-n-q}+a_{1} b_{r-n-1} & & a_{1-n} b_{r-1}+\sum_{q=2-n}^{0} a_{q} b_{r-n-q} .
\end{array}
$$

That is,

$$
\begin{gathered}
c_{n-r+1,1}=\sum_{q=r-n}^{r-1} a_{q} b_{r-n-q}=\sum_{q=r-n-1}^{r-2} a_{q} b_{r-n-q}=c_{n-r+2,2}, \\
c_{n-r+2,2}=\sum_{q=r-n-1}^{r-2} a_{q} b_{r-n-q}=\sum_{q=r-n-2}^{r-3} a_{q} b_{r-n-q}=c_{n-r+3,3}, \\
\ldots \quad \ldots \quad \cdots \\
c_{n-1, r-1}=\sum_{q=2-n}^{1} a_{q} b_{r-n-q}=\sum_{q=1-n}^{0} a_{q} b_{r-n-q}=c_{n, r} .
\end{gathered}
$$

Thus, the entries down the lower- $(n-r)$ th diagonal of $C$ are equal for $r=1,2, \ldots, n-1$.

Therefore, $A B$ is also a Toeplitz matrix. Furthermore, if we let $A B=C=\left(c_{j-i}\right)_{i, j=1}^{n}$, then

$$
c_{i}=c_{n-i, n}=\sum_{q=i-n+1}^{i} a_{q} b_{i-q}=a_{i} b_{0}+a_{0} b_{i}+\sum_{\substack{q=i-n+1 \\ q \neq 0}}^{i-1} a_{q} b_{i-q}
$$

and

$$
c_{i-n}=c_{n-i+1,1}=\sum_{q=i-n}^{i-1} a_{q} b_{i-n-q}=a_{i-n} b_{0}+a_{0} b_{i-n}+\sum_{\substack{q=i-n+1 \\ q \neq 0}}^{i-1} a_{q} b_{i-n-q} .
$$

Hence,

$$
\begin{aligned}
c_{i} a_{j-n} & =b_{0} a_{i} a_{j-n}+a_{0} b_{i} a_{j-n}+\sum_{\substack{q=i-n+1 \\
q \neq 0}}^{i-1} a_{q} b_{i-q} a_{j-n} \\
& =b_{0} a_{i-n} a_{j}+a_{0} b_{i-n} a_{j}+\sum_{\substack{q=i-n+1 \\
q \neq 0}}^{i-1} a_{q} b_{i-q-n} a_{j}=c_{i-n} a_{j}
\end{aligned}
$$

and

$$
\begin{aligned}
c_{i} b_{j-n} & =b_{0} a_{i} b_{j-n}+a_{0} b_{i} b_{j-n}+\sum_{\substack{q=i-n+1 \\
q \neq 0}}^{i-1} a_{q} b_{i-q} b_{j-n} \\
& =b_{0} a_{i-n} b_{j}+a_{0} b_{i-n} b_{j}+\sum_{\substack{q=i-n+1 \\
q \neq 0}}^{i-1} a_{q} b_{i-q-n} b_{j}=c_{i-n} b_{j}
\end{aligned}
$$

for any $i, j=1,2, \ldots, n-1$.

Next we obtain a sufficient condition for a Toeplitz matrix to have Toeplitz positive integer powers by the lemma above, and clarify the connections between the entries of the Toeplitz matrix and those of its positive integer powers. 
Lemma 2 Let $A=\left(a_{j-i}\right)_{i, j=1}^{n}$ be a Toeplitz matrix, $n \geq 2$. If $a_{i} a_{j-n}=a_{i-n} a_{j}$ for $i, j=1,2, \ldots, n-1$, then $A^{k}$ is also a Toeplitz matrix for any positive integer $k$. Furthermore, if we let $A^{k}=\left(a_{j-i}^{(k)}\right)$, then $a_{i} a_{j-n}^{(k)}=a_{i-n} a_{j}^{(k)}$ for $i, j=1,2, \ldots, n-1$.

Proof. If $A$ is a scalar matrix, then the lemma holds naturally. Otherwise, We prove by induction on $k$. The case $k=1$ holds naturally and the case $k=2$ is proved by choosing $B=A$ in Lemma 1 .

Now we suppose that the lemma holds for $k-1$. Since $A^{k}=A A^{k-1}$, the lemma holds by taking $B=A^{k-1}$ in Lemma 1.

Now we derive a necessary condition for Toeplitz matrices to have Toeplitz squares.

Lemma 3 Let $A=\left(a_{j-i}\right)_{i, j=1}^{n}$, and $A^{2}$ be Toeplitz matrices, $n \geq 2$. Then $a_{i} a_{j-n}=a_{i-n} a_{j}$ for $i, j=1,2, \ldots, n-1$.

Proof. Suppose that $A=\left(a_{j-i}\right)_{i, j=1}^{n}, A^{2}=\left(c_{j-i}\right)_{i, j=1}^{n}$. Then for $r=1,2, \ldots, n-2$, the entries of $A^{2}$ down the upper-rth diagonal are equal:

$$
\sum_{q=0}^{n-1} a_{q} a_{r-q}=\sum_{q=-1}^{n-2} a_{q} a_{r-q}=\cdots=\sum_{q=1-n+r}^{r} a_{q} a_{r-q} ;
$$

the entries of $A^{2}$ down the lower- $(n-r)$ th diagonal are equal:

$$
\sum_{q=r-n}^{r-1} a_{q} a_{r-n-q}=\sum_{q=r-n-1}^{r-2} a_{q} a_{r-n-q}=\cdots=\sum_{q=1-n}^{0} a_{q} a_{r-n-q} .
$$

And the entries of $A^{2}$ on the main diagonal are also equal:

$$
c_{0}=\sum_{q=0}^{n-1} a_{q} a_{-q}=\sum_{q=-1}^{n-2} a_{q} a_{-q}=\cdots=\sum_{q=1-n}^{0} a_{q} a_{-q} .
$$

Compare both sides of each "=", we derive $(n-1)^{2}$ identities: $a_{i} a_{j-n}=a_{i-n} a_{j}$ for $i, j=1,2, \ldots, n-1$.

Finally, our main theorem of this paper can be easily obtained.

Theorem 4 Let $A=\left(a_{j-i}\right)_{i, j=1}^{n}$ be a Toeplitz matrix, $n \geq 2$. Then any positive integer power of $A$ is also a Toeplitz matrix if and only if $a_{i} a_{j-n}=a_{i-n} a_{j}$ for $i, j=1,2, \ldots, n-1$.

Proof. The sufficiency is proved by Lemma 2 , and the necessity is proved by Lemma 3 .

The condition in Theorem 4 can be examined by computer easily, so it is a valuable condition for us to study the powers of Toeplitz matrices. We note that circulant matrices are well-known examples of such Toeplitz matrices satisfying the necessary and sufficient condition above.

\section{Examples}

Now we give some matrices, and compare the powers of them.

$$
\begin{array}{rlrl}
A & =\left(\begin{array}{llll}
1 & 2 & 3 & 4 \\
4 & 1 & 2 & 3 \\
3 & 4 & 1 & 2 \\
2 & 3 & 4 & 1
\end{array}\right), & A^{5}=\left(\begin{array}{llll}
25056 & 25072 & 24928 & 24944 \\
24944 & 25056 & 25072 & 24928 \\
24928 & 24944 & 25056 & 25072 \\
25072 & 24928 & 24944 & 25056
\end{array}\right) \\
B=\left(\begin{array}{llll}
1 & 2 & 4 & 8 \\
4 & 1 & 2 & 4 \\
2 & 4 & 1 & 2 \\
1 & 2 & 4 & 1
\end{array}\right), & B^{5}=\left(\begin{array}{llll}
28361 & 34266 & 40476 & 47576 \\
23788 & 28361 & 34266 & 40476 \\
20238 & 23788 & 28361 & 34266 \\
17133 & 20238 & 23788 & 28361
\end{array}\right) \\
C=\left(\begin{array}{llll}
1 & 2 & 4 & 0 \\
4 & 1 & 2 & 4 \\
2 & 4 & 1 & 2 \\
1 & 2 & 4 & 1
\end{array}\right), & C^{5}=\left(\begin{array}{llll}
10681 & 11674 & 12988 & 9520 \\
14668 & 16841 & 18394 & 12988 \\
13214 & 14668 & 16841 & 11674 \\
11997 & 13214 & 14668 & 10681
\end{array}\right)
\end{array}
$$


The matrix $A$ is a circulant matrix, hence any positive integer power of $A, A^{5}$ for example, is still a Toeplitz matrix (a circulant matrix actually) by Theorem 4 . The matrices $B$ and $C$ are both Toeplitz matrices, however, $B^{5}$ is a Toeplitz matrix while $C^{5}$ is no longer a Toeplitz matrix since $C=\left(c_{j-i}\right)_{i, j=1}^{4}$ does not satisfy the conditions in Theorem 4: $c_{3} c_{2-4}=0 \times 2 \neq c_{3-4} c_{2}=4 \times 4$.

Toeplitz matrices have nice properties and great applications in science and industry, there remain many problems for us to study.

\section{References}

Böttcher, A., \& Silbermann, B. (1999). Introduction to Large Truncated Toeplitz Matrices. New York, NY: Springer.

Chan, R., \& Ng, M. (1996). Conjugate gradient methods for Toeplitz systems. SIAM Review, 38(3), 427-482. http://dx.doi.org/10.1137/S0036144594276474

Gray, R. M. (2006). Toeplitz and circulant matrices: A review. Now Pub.

Grenander, U., \& Szegö, G. (1958). Toeplitz Forms and Their Applications. Berkeley and Los Angeles: University of California Press.

Greville, T. N. E. (1983). Toeplitz matrices with Toeplitz inverses. Linear Algebra and its Applications, 55, 877-92. http://dx.doi.org/10.1016/0024-3795(83)90168-4

Horn, R. A., \& Johnson, C. R. (1985). Matrix Analysis. England: Cambridge University Press.

Kailath, T., \& Sayed, A. (1995). Displacement structure: theory and applications. SIAM Review, 37(3), 297-386. http://dx.doi.org/10.1137/1037082

Shalom, T. (1987). On Algebras of Toeplitz Matrices. Linear Algebra and its Applications, 96, 211-226. http://dx.doi.org/10.1016/0024-3795(87)90345-4

\section{Copyrights}

Copyright for this article is retained by the author(s), with first publication rights granted to the journal.

This is an open-access article distributed under the terms and conditions of the Creative Commons Attribution license (http://creativecommons.org/licenses/by/3.0/). 